\title{
Review Article \\ Feasibility and Safety of Single-Incision Laparoscopic Surgery in Gastric Benign and Malignant Diseases
}

\author{
Kong Jing, Wu Shuo-dong, Fan Ying, and Wang Yu \\ Department of Minimally Invasive Surgery, Shengjing Hospital, China Medical University, Sanhao Street No. 36, \\ Shenyang, Liaoning 110004, China
}

Correspondence should be addressed to Wu Shuo-dong; yibeishui2@foxmail.com

Received 13 March 2014; Accepted 3 May 2014; Published 28 May 2014

Academic Editor: Antoni Castells

Copyright (C) 2014 Kong Jing et al. This is an open access article distributed under the Creative Commons Attribution License, which permits unrestricted use, distribution, and reproduction in any medium, provided the original work is properly cited.

\begin{abstract}
We systematically searched Medline and Cochrane Library and the related references occurred in the citations until February 2013 and included all the cases who suffered from gastric benign and malignant diseases where single incision laparoscopic surgery was performed. A total of 69 cases with gastric diseases undergoing SILS surgery were reviewed to evaluate the feasibility, safety, and potential benefits of SILS for the gastric diseases. Demographic data, morphologic characterisation of the lesion, and perioperative parameters were analyzed retrospectively. The initial experience with SILS for benign gastric diseases and early gastric cancer showed that it is feasible and safe when performed by experienced laparoscopic surgeons. Despite the limited number and technical difficulties it can be proposed in selected patients.
\end{abstract}

\section{Introduction}

Laparoscopic gastrectomy is being accepted as a safe and feasible surgical procedure for a wide range of gastric benign diseases owing to its less invasive and comparable oncologic outcomes. Since the first laparoscopy-assisted distal gastrectomy reported by Kitano et al. in 1994 [1], laparoscopic radical gastrectomy (LADG) for early gastric cancer has also become a widely accepted procedure. Some randomized control studies (RCTs) have clearly demonstrated short-term benefits including early postoperative recovery, decreased postoperative pain, shorter hospitalization, and similar number of harvested lymph nodes [2-5]. Laparoscopic surgery typically uses three or four ports for gastric procedure, with more potential morbidity from port-site bleeding, hernia, and infection. In recent years, laparoscopic surgery has become even less invasive with the introduction of transumbilical single-incision surgery due to its cosmetic advantage. It has been used for various cholecystectomies, appendectomies, nephrectomies, and other related surgical operations, and recently this technique has been applied in gastric surgery. Because of the limited number of studies reported about SILS in gastric diseases, its clinical significance remains to be elucidated. The purpose of this review is to assess the feasibility and safety of SILS for gastric diseases.

\section{Materials and Methods}

2.1. Literature Search Strategies. A systematic search of the scientific literature was carried out using the online databases (PubMed and the Cochrane library) and the related references that occurred in the citations to identify any articles missed by the initial search were manually searched up to February 1, 2013. The search terms were "single incision," "single port," "single access," "single site," "laparoscop*," "laparoendoscop"," "peritoneoscop*," "stomoch*," "gastric," "gastrectomy," and "gastro*." There were no language restrictions during the search and the search strategy in this study was listed as below:

\#14 Add Search (((\#13) OR \#12) OR \#11) OR \#10

\#13 Add Search gastro*

\#12 Add Search gastrectomy

\#11 Add Search gastric

\#10 Add Search stomoch*

\#9 Add Search ((\#8) OR \#7) OR \#6

\#8 Add Search peritoneoscop*

\#7 Add Search laparoendoscop*

\#6 Add Search laparoscop*

\#5 Add Search (((\#4) OR \#3) OR \#2) OR \#1

\#4 Add Search single site

\#3 Add Search single access 
\#2 Add Search single port

\#1 Add Search single incision.

2.2. Inclusion Criteria. Articles were selected if the abstract contained data of patients who underwent SILS for gastric diseases in all the forms, such as RCTs, other controlled or retrospective studies, and case reports. The references of these articles were also reviewed in order to find additional relevant studies. Only the latest and most complete study was included when the duplicate cases in different publications were reviewed. Reports with animal model in SILS, review articles, and studies about different SILS for the treatment of obesity were excluded from this study. The full text provided that the data for this review were verified by each of the two investigators (JK and YF) who independently decided on inclusion/exclusion and independently abstracted the study data. Disagreements and discrepancies were resolved by consensus.

\section{Results}

Up to February 1, 2013, a total of 69 cases with gastric diseases underwent SILS surgery. Among these, 7 studies with 31 patients undergoing SILS partial resection of the stomach or gastric wedge resection were reviewed. SILS subtotal gastrectomy was performed in 15 patients introduced by 5 studies, respectively. And in 4 of these 5 studies, the lymphadenectomy was also performed for 14 patients with early gastric cancer. A case about SILS perforated gastric ulcer repair was reported. Furthermore, the method of intracorporeal circular-stapled Billroth I anastomosis for 20 cases that underwent SILS distal gastrectomy was introduced in 1 study. Another method for intracorporeal anastomosis for gastrojejunostomy was performed in 2 cases with gastric outlet obstruction.

3.1. SILS in Partial Gastrectomy for Submucosal Tumors. A total of 7 studies with 31 patients undergoing SILS partial resection of the stomach or gastric wedge resection were reviewed [6-12] (Tables 1 and 2). In Na et al's report [6], 7 patients diagnosed as gastric submucosal tumor underwent the intragastric wedge resection. Among these 31 cases, 27 GISTs [6-12], 1 nonspecific inflammatory [12], 2 leiomyoma [6], and 1 benign carcinoma tumor [11] were confirmed by the final pathology. 11 tumors were located on the fundus of stomach $[6,7,12], 8$ tumors located on the body of the stomach $[6,7,11,12], 4$ tumors located on the anterior wall $[7,8,11], 3$ tumor located on the posterior wall [7, $9]$, and 5 tumors located on the gastric cardia $[6,10]$. Pathology confirmed the diameter of tumors from $0.6 \mathrm{~cm}$ to $5.4 \mathrm{~cm}(<2 \mathrm{~cm}$ in 5 cases and $\geq 2 \mathrm{~cm}$ in 26 cases $)$. Though flexible laparoscope and curved or double-bended graspers were used frequently and made the SILS operation more easily, conventional laparoscopic instruments can achieve the similar clinical outcomes [7]. Also an extra mini-port for $2 \mathrm{~mm}$ mini-loop was often used to retract the liver or gastric wall surrounding the tumor to facilitate the dissection and resection [8-11]. Ranges of all the operative times were from
64 to 147 minutes and estimated blood losses were minimal. There were no cases converted to conventional (multiport) laparoscopic or open operations in all the above patients. Except wounding bleeding in one case [6], no intraoperative or other postoperative complications were experienced in all the patients including anastomotic leakage, bleeding, or anastomotic stenosis.

3.2. SILS in Distal Gastrectomy for Peptic Ulcer and Early Gastric Cancer. SILS subtotal gastrectomy was performed in 15 patients introduced by 5 studies, respectively [13-17]. And in 4 studies, the lymphadenectomy was also performed for 14 patients with early gastric cancer [13-16] (Tables 3 and 4).

A patient with an intractable gastric ulcer was treated by SILS gastrectomy with intracorporeal Roux-en-Y reconstruction [17]. In this patient, a $3 \mathrm{~cm}$ hernial orifice was seen between the duodenum and the retroperitoneal area. A large opening was made in the hernial orifice by mobilizing the right-side colon. The appendix was also excised is this patient. The operative time was $412 \mathrm{~min}$, with blood loss of $90 \mathrm{~mL}$. An additional $2 \mathrm{~mm}$ mini-loop retractor was inserted through an extra incision in the right subcostal space to create the operative field. The patient started a normal diet on day 3 and was discharged on postoperative day 14 with no complications.

14 patients underwent single-incision laparoscopic distal gastrectomy and lymph node $\mathrm{D} 1 \alpha$ or $\mathrm{D} 1 \beta$ or $\mathrm{D} 2$ dissection in 4 reports [13-16]. Computed tomography showed that there was no regional LN or distant metastases before the operation. The final pathology report showed that the tumor had invaded the submucosa or proper muscle and with no seromuscular layer infiltration. A total of 236 lymph nodes were retrieved. Except one patient with 5 metastases among dissected 21 LNs [14] and one patient with 1 metastasis [16], lymph nodes of other patients were identified with no tumor metastasis. Proximal and distal resection margins were free of tumor. Gastroduodenostomy was performed in 8 patients $[13,14]$ and 5 cases with gastrojunostomy $[15,16]$ and 1 case with Roux-en-Y reconstruction [13]. The range of the operative time was from $140 \mathrm{~min}$ to $385 \mathrm{~min}$. And the blood loss was less than $150 \mathrm{~mL}$ in all patients. No major complications were observed including anastomotic leakage, stenosis, or hemorrhage. Although surgical-site infection was shown in 2 cases and stasis in one case [13], all recovered with conservative treatment. $2 \mathrm{~mm}$ mini-loop retractor $[13,14]$, flexible laparoscope $[13,15]$, and curved instruments $[14,15]$ were used in most of them.

3.3. SILS in Other Reports with Gastric Diseases. A case about SILS perforated gastric ulcer repair was reported [18]. The operation lasted 108 minutes. The blood loss was $86 \mathrm{~min}$. No additional trocars needed to be inserted and flexible laparoscope and curved reusable instruments were used in this patient.

Furthermore, the method of intracorporeal circularstapled Billroth I anastomosis for 20 cases that underwent SILS distal gastrectomy was introduced in 1 study [19]. The average operative time was 279 (208 319) min. Neither 


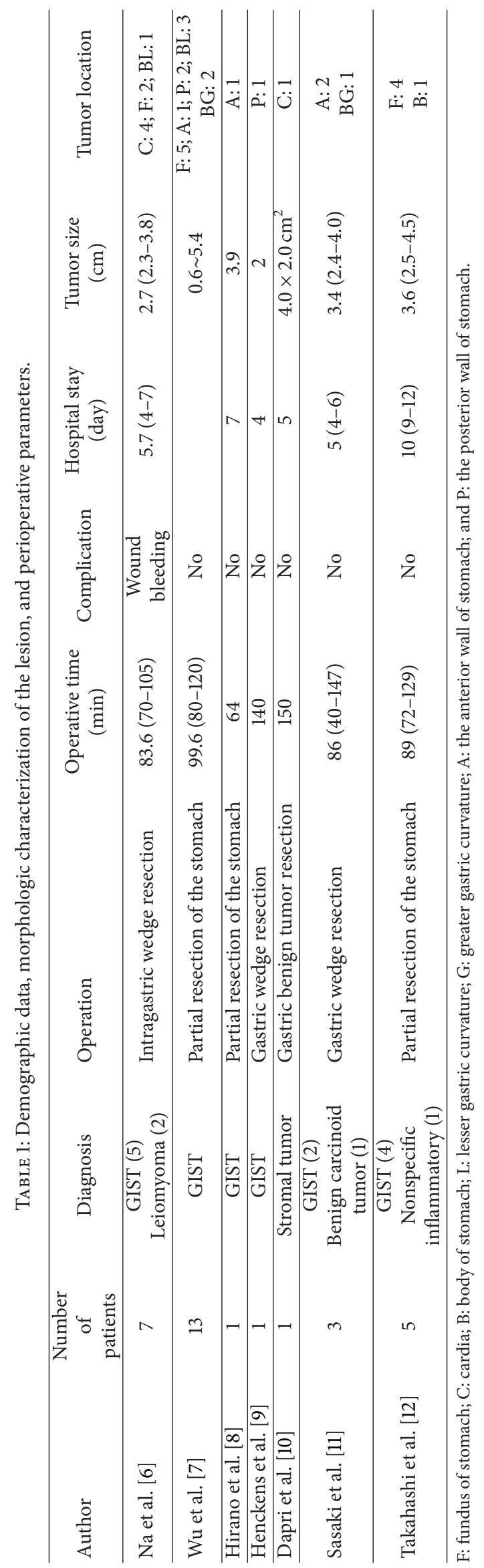




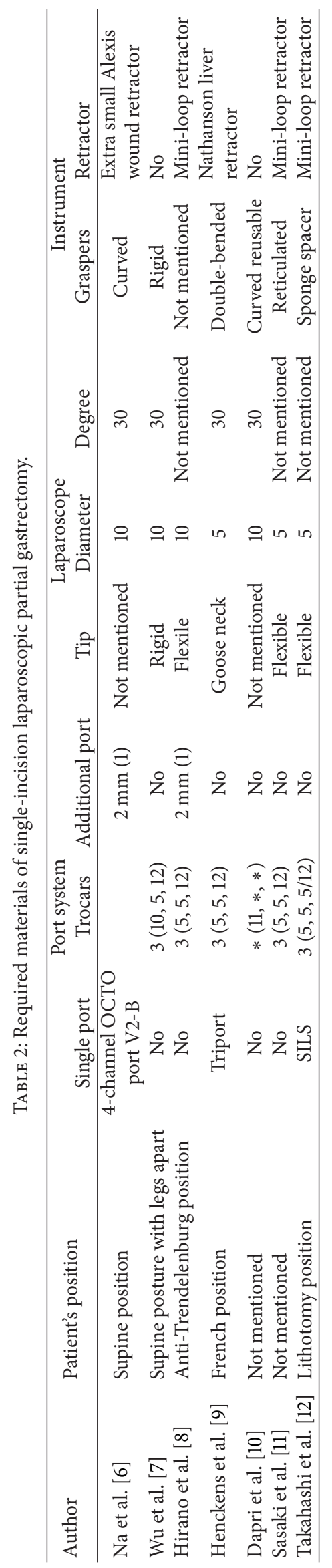




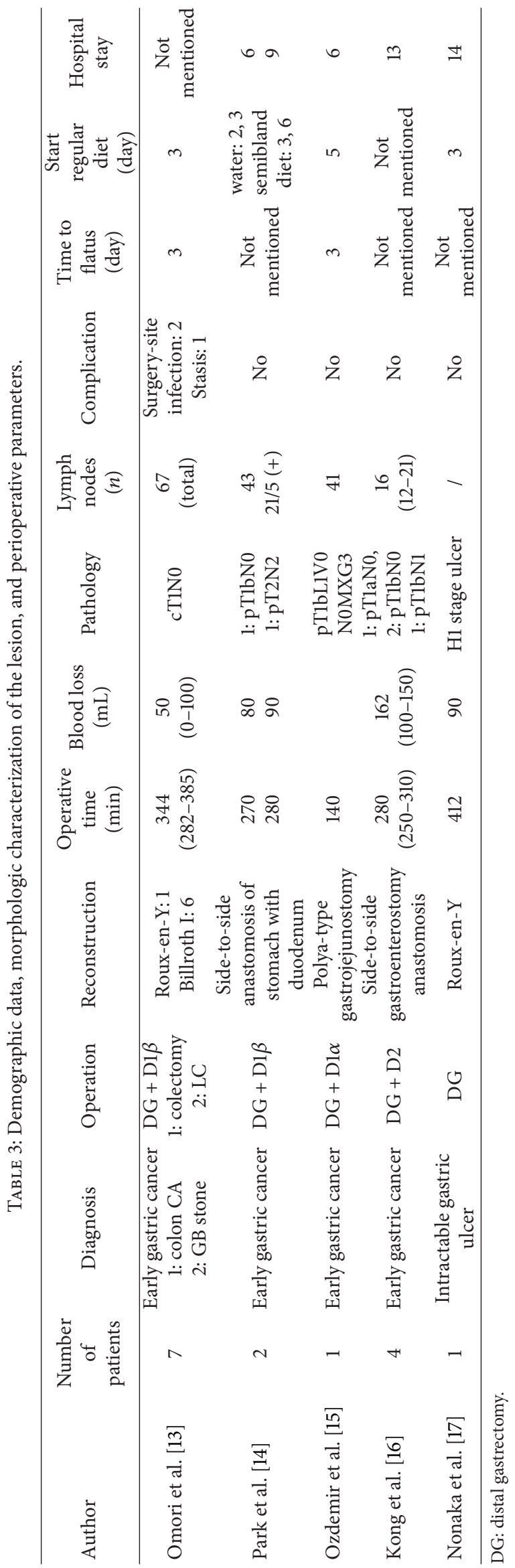




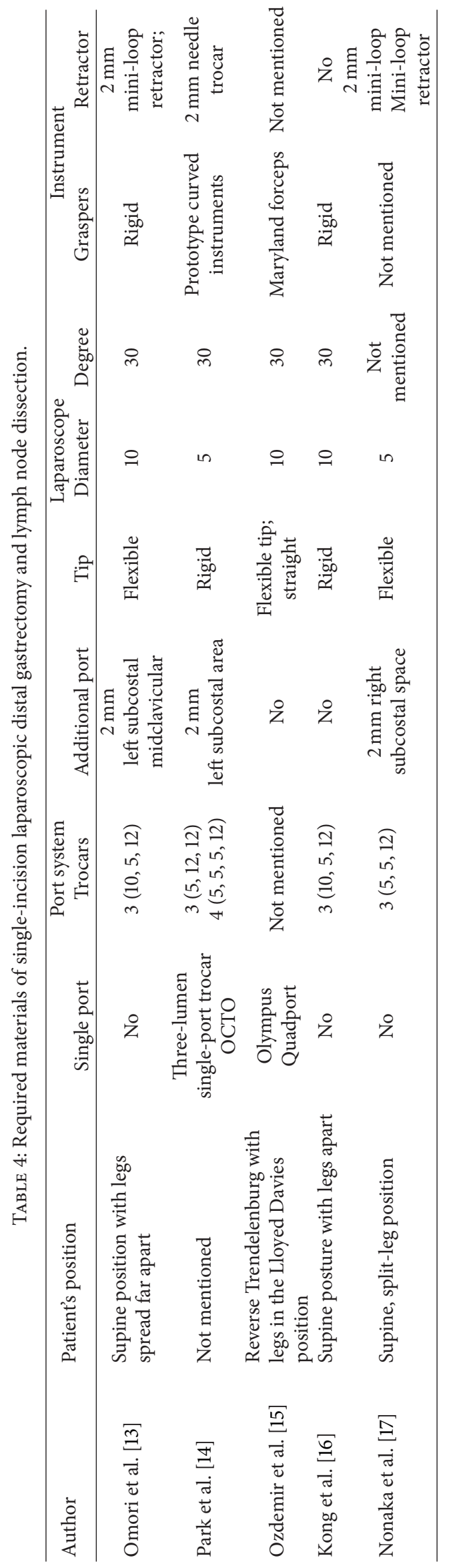


postoperative complications, including anastomotic leakage and stricture, nor postoperative mortality was observed in this study.

Another method for intracorporeal anastomosis for gastrojejunostomy was performed in 2 cases with gastric outlet obstruction [20].

There were no cases converted to conventional (multiport) laparoscopic or open operations in all above patients.

\section{Discussion}

Since the introduction of laparoscopy in 1910 [21], the benefits of minimally invasive surgery have expanded its applicability to the treatment of benign and malignant gastric diseases [22]. Many researches have suggested that the laparoscopic approach to gastric GISTs or other benign gastric diseases is safe and effective, resulting in a short hospital stay duration and low morbidity $[23,24]$. Although the radicalness and safety of laparoscopic tumor resection are controversial, there have been some retrospective studies which confirmed that laparoscopic radical gastrectomy for gastric cancer can achieve the same effect with open operation $[3,4,25]$; even laparoscopic operation is superior to the traditional operation in the matter of postoperative hospital stay, infection of incision, and postoperative complications [5, 26-28].

SILS was described by Pelosi in 1992; a single-puncture laparoscopic appendectomy was performed successfully [29]. With the popularization and development of laparoscopic technology in recent years, the application of SILS has become the current clinical research hot topic. The potential advantages of this approach are always related to the scarless healing of the incision and less wound-related complications, including bleeding, infection, and hernia.

But since the first publication about single-incision laparoscopic sleeve gastrectomy reported by Saber et al. [30], only few sporadic reports about single-incision laparoscopic gastric surgery have appeared worldwide. As the result of this review showed, only a total of 31 patients in 7 studies underwent SILS partial resection of the stomach or gastric wedge resection for gastric submucosal lesions. Simple SILS subtotal gastrectomy and perforated gastric ulcer repair were performed in 1 patient with gastric ulcer, respectively. And SILS subtotal gastrectomy with lymphadenectomy was also performed for 14 patients with early gastric cancer. Though the number of the cases was small and only the sporadic case reports were reported and reviewed, the successful SILS gastric operation itself was exciting.

SILS partial resection of the stomach or gastric wedge resection was well performed in these reports with no perioperative complications. The large volume and the quite mobile stomach made it easier. When the greater and lesser curvatures have been freed from surrounding attachments, the submucosal lesions can be quickly and accurately located especially by intraoperative endoscopy. And they also can be resected easily by Endo-GIA. Ranges of operative times were from 64 to 147 minutes and estimated blood losses were minimal. Although factors affecting operative time including the location and size of the tumor were not controlled for in Otani's study, there was no significant difference in operative time between SILS ( $89 \mathrm{~min}$ ) and Otani et al. previously reported operative time for conventional laparoscopy (131 min) [31].

Also the patients were selected based on the tumor size, location, and growth morphology. For the submucosal benign diseases, the lesion's location was limited at the body of the stomach. When the lesion located at the cardia increased the difficulty of the operation, intragastric wedge resection by SILS was performed [6]. In Henckens et al's preliminary experience with less partial gastrectomy [9], larger or full-thickness tumors in close proximity to the pylorus often require a more formal resection (i.e., antrectomy and gastrojejunostomy) due to the high probability that a wedge resection will result in the narrowing of the distal stomach, causing iatrogenic gastric outlet obstruction. Masses in the proximity of the gastroesophageal junction can be managed similarly to tumors near the pylorus. It needs to be said that the resection of a tumor at these anatomic sites would be more difficult by laparoscopic single-site surgery. And a conventional laparoscopy or even open resection in these cases should be considered.

Adequate lymph node dissection is a complex and highly difficult operation in radical gastrectomy with gastric cancer. When performed by SILS, the difficulties appreciably increased owing to the conflict between the laparoscope and instruments inserted through the same single-incision and the lack of the operative field exposure with assistance. In early gastric cancer, lymph node involvement is infrequent and when present it predominantly involves perigastric nodes. There is, therefore, current acceptance of laparoscopic resection for early gastric cancer. Fourteen patients diagnosed as gastric early cancer were confirmed by preoperative evaluation and final pathology. Despite the longer operative time needed, there were no serious complications in these clinical reports. The number of harvested lymph nodes for malignant cases was from 12 to 67 and surgical margins were negative in all cases. Although the fewer cases and the short observation time still needed to further evaluate the benefits of this new minimally invasive technique, it is feasible and safe when performed by experienced laparoscopic surgeons.

No major short-term complications were observed including anastomotic leakage, stenosis, or hemorrhage in all these patients. Wounding bleeding was shown in one case with SILS partial gastrectomy [6] and surgical-site infection in 2 cases and stasis in one case with SILS distal gastrectomy [13]; all these patients recovered with conservative treatment. Unfortunately, no comparative studies were found in this review to compare the short-term and longterm complications with conventional laparoscopic surgery. In Lee et al.'s [32] report, the porcine model was used to compare the perioperative outcomes of SILS and conventional laparoscopic distal gastrectomy with $\mathrm{D} 1+\mathrm{b}$ lymph node dissection. The single-incision laparoscopic group was associated with a significantly longer operation time, but it had a similar mean number of resected lymph nodes and with similar inflammatory reaction and complication rates when compared with conventional laparoscopic. A recent meta-analysis demonstrated that single-incision laparoscopic 
cholecystectomy is a safe procedure for the treatment of uncomplicated gallstone disease, with postoperative outcome similar to that of standard multiport laparoscopic cholecystectomy [33]. Chakravartty et al. [34] compared 111 patients that underwent SILS-AGB (adjustable gastric banding) and 99 patients that underwent LAGB and reported that in the SILS-AGB group the median operating time $(70 \mathrm{~min})$ was not significantly longer than in the LAGB group $(61.5 \mathrm{~min}$, $P=0.07)$. However, SILS-AGB patients used less opiates $(P<0.01)$ than the LAGB patients. There was no difference in morbidity, mortality, or readmission rates.

However, three trocars at the vertex of inverted equilateral triangle about $10 \mathrm{~mm}$ apart may avoid collision with each other slightly. The laparoscope with optical flexible 30 degrees, flexible or curved instruments, a $2 \mathrm{~mm}$ miniloop retractor, and an additional $2 \mathrm{~mm}$ subcostal port were helpful to facilitate surgery SILS and decrease operative time. Auxiliary traction sutures and intra-abdominal retractors can make up the shortage and expose the operative field clearly as much as possible.

In conclusion, the initial experience with single-incision laparoscopic partial gastrectomy or radical gastrectomy for gastric benign and early malignant diseases is feasible and safe when performed by experienced laparoscopic surgeons. As a result of the fewer cases and the short observation time in this group, it is still needed to further expand the number of the case and extend the follow-up time to determine the benefits of this new minimally invasive technique.

\section{Conflict of Interests}

None of the authors have any potential conflict of interests or any financial interests.

\section{Authors' Contribution}

Wu Shuo-dong designed the study, conducted statistical analysis, and approved the final version of the paper. Kong Jing collected and verified data, prepared the entire initial draft including the structure of the paper, use of endnote for references, and covering letter, and prepared the final version of the paper after all the corrections. Fan Ying collected and verified data. Wang Yu collected the data.

\section{References}

[1] S. Kitano, Y. Iso, M. Moriyama, and K. Sugimachi, "Laparoscopy-assisted Billroth I gastrectomy," Surgical Laparoscopy and Endoscopy, vol. 4, no. 2, pp. 146-148, 1994.

[2] S. H. Noh, W. J. Hyung, and J. H. Cheong, "Minimally invasive treatment for gastric cancer: Approaches and selection process," Journal of Surgical Oncology, vol. 90, no. 3, pp. 188-193, 2005.

[3] M. Ohgami, Y. Otani, K. Kumai, T. Kubota, Y. I. Kim, and M. Kitajima, "Curative laparoscopic surgery for early gastric cancer: five years experience," World Journal of Surgery, vol. 23, no. 2, pp. 187-192, 1999.

[4] M. C. Kim, K. H. Kim, H. H. Kim, and G. J. Jung, "Comparison of laparoscopy-assisted by conventional open distal gastrectomy and extraperigastric lymph node dissection in early gastric cancer," Journal of Surgical Oncology, vol. 91, no. 1, pp. 90-94, 2005.

[5] M. C. Kim, H. H. Kim, and G. J. Jung, "Surgical outcome of laparoscopy-assisted gastrectomy with extraperigastric lymph node dissection for gastric cancer," European Journal of Surgical Oncology, vol. 31, no. 4, pp. 401-405, 2005.

[6] J. U. Na, S. I. Lee, and S. M. Noh, "The single incision laparoscopic intragastric wedge resection of gastric submucosal tumor," Journal of Gastric Cancer, vol. 11, no. 4, pp. 225-229, 2011.

[7] S. D. Wu, J. Kong, Y. Su et al., "Safety and application of transumbilical single-incision laparoscopic gastrectomy for GIST: SILS in benign gastric disease," Surgical Innovation, vol. 20, pp. 365-369, 2013.

[8] Y. Hirano, T. Watanabe, T. Uchida, S. Yoshida, H. Kato, and O. Hosokawa, "Laparoendoscopic single site partial resection of the stomach for gastrointestinal stromal tumor," Surgical Laparoscopy, Endoscopy and Percutaneous Techniques, vol. 20, no. 4, pp. 262-264, 2010.

[9] T. Henckens, D. van de Putte, K. van Renterghem, W. Ceelen, P. Pattyn, and Y. van Nieuwenhove, "Laparoendoscopic singlesite gastrectomy for a gastric GIST using double-bended instruments," Journal of Laparoendoscopic and Advanced Surgical Techniques, vol. 20, no. 5, pp. 469-471, 2010.

[10] G. Dapri, R. Ntounda, J. Himpens, P. Carnevali, S. Scomersi, and G.-B. Cadière, "Single-incision transumbilical laparoendoscopic gastric benign tumor resection," Annals of surgical oncology, vol. 18, no. 1, p. 191, 2011.

[11] A. Sasaki, K. Koeda, J. Nakajima, T. Obuchi, S. Baba, and G. Wakabayashi, "Single-incision laparoscopic gastric resection for submucosal tumors: report of three cases," Surgery Today, vol. 41, no. 1, pp. 133-136, 2011.

[12] T. Takahashi, H. Takeuchi, H. Kawakubo, Y. Saikawa, N. Wada, and Y. Kitagawa, "Single-incision laparoscopic surgery for partial gastrectomy in patients with a gastric submucosal tumor," The American Surgeon, vol. 78, no. 4, pp. 447-450, 2012.

[13] T. Omori, T. Oyama, H. Akamatsu, M. Tori, S. Ueshima, and T. Nishida, "Transumbilical single-incision laparoscopic distal gastrectomy for early gastric cancer," Surgical Endoscopy and Other Interventional Techniques, vol. 25, no. 7, pp. 2400-2404, 2011.

[14] D. J. Park, J. H. Lee, S.-H. Ahn, A. K. H. Eng, and H.-H. Kim, "Single-port laparoscopic distal gastrectomy with $\mathrm{D} 1+\beta$ lymph node dissection for gastric cancers: Report of 2 cases," Surgical Laparoscopy, Endoscopy and Percutaneous Techniques, vol. 22, no. 4, pp. e214-e216, 2012.

[15] B. A. Ozdemir, R. L. Thomas, and Y. Soon, "Single-port laparoscopic subtotal gastrectomy with DI $\alpha$ lymphadenectomy," Surgical Innovation, vol. 18, no. 4, pp. NP1-NP4, 2011.

[16] J. Kong, S.-D. Wu, and Y. Su, "Translumenal single-incision laparoscopy radical gastrectomy with $\mathrm{d} 2$ lymph node dissection for early gastric cancer-primary experience with less invasive surgery in China," Journal of Laparoendoscopic and Advanced Surgical Techniques, vol. 23, no. 2, pp. 141-145, 2013.

[17] T. Nonaka, S. Hidaka, A. Takafumi et al., "Single-incision laparoscopy-assisted subtotal gastrectomy for intractable gastric ulcer: a case report," Surgical Laparoscopy, Endoscopy and Percutaneous Techniques, vol. 22, no. 4, pp. e210-e213, 2012.

[18] G. Dapri, H. E. Mourad, J. Himpens, G. Evola, L. Marsili, and G.-B. Cadière, "Transumbilical single-access laparoscopic perforated gastric ulcer repair," Surgical Innovation, vol. 19, no. 2, pp. 130-133, 2012. 
[19] T. Omori, K. Tanaka, M. Tori, S. Ueshima, H. Akamatsu, and T. Nishida, "Intracorporeal circular-stapled Billroth i anastomosis in single-incision laparoscopic distal gastrectomy," Surgical Endoscopy and Other Interventional Techniques, vol. 26, no. 5, pp. 1490-1494, 2012.

[20] P. Bucher, F. Pugin, and P. Morel, “Transumbilical singleincision laparoscopic intracorporeal anastomosis for gastrojejunostomy: Case report," Surgical Endoscopy and Other Interventional Techniques, vol. 23, no. 7, pp. 1667-1670, 2009.

[21] C. Nezhat, W. K. Winer, J. D. Cooper, F. Nezhat, and C. Nezhat, "Endoscopic infertility surgery," Journal of Reproductive Medicine for the Obstetrician and Gynecologist, vol. 34, no. 2, pp. 127-134, 1989.

[22] V. Liew, C. Taylor, M. Ghusn, M. Jamnagerwalla, and L. Layani, "Laparoscopic gastric resection for benign and malignant conditions: lessons learned from 35 consecutive cases," ANZ Journal of Surgery, vol. 77, no. 9, pp. 787-791, 2007.

[23] R. P. DeMatteo, J. J. Lewis, D. Leung, S. S. Mudan, J. M. Woodruff, and M. F. Brennan, "Two hundred gastrointestinal stromal tumors: Recurrence patterns and prognostic factors for survival," Annals of Surgery, vol. 231, no. 1, pp. 51-58, 2000.

[24] K. L. Huguet, R. M. Rush Jr., D. J. Tessier et al., "Laparoscopic gastric gastrointestinal stromal tumor resection: the Mayo Clinic experience," Archives of Surgery, vol. 143, no. 6, pp. 587590, 2008.

[25] S. H. Noh, W. J. Hyung, and J. H. Cheong, "Minimally invasive treatment for gastric cancer: approaches and selection process," Journal of Surgical Oncology, vol. 90, no. 3, pp. 188-193, 2005.

[26] J. M. Park, S. H. Jin, S. R. Lee et al., "Complications with laparoscopically assisted gastrectomy: multivariate analysis of 300 consecutive cases," Surgical Endoscopy and Other Interventional Techniques, vol. 22, no. 10, pp. 2133-2139, 2008.

[27] K. W. Ryu, Y. W. Kim, J. H. Lee et al., "Surgical complications and the risk factors of laparoscopy-assisted distal gastrectomy in early gastric cancer," Annals of Surgical Oncology, vol. 15, no. 6, pp. 1625-1631, 2008.

[28] B. Topal, E. Leys, N. Ectors, R. Aerts, and F. Penninckx, "Determinants of complications and adequacy of surgical resection in laparoscopic versus open total gastrectomy for adenocarcinoma," Surgical Endoscopy and Other Interventional Techniques, vol. 22, no. 4, pp. 980-984, 2008.

[29] M. A. Pelosi and M. A. Pelosi III, "Laparoscopic appendectomy using a single umbilical puncture (minilaparoscopy)," Journal of Reproductive Medicine for the Obstetrician and Gynecologist, vol. 37, no. 7, pp. 588-594, 1992.

[30] A. A. Saber, M. H. Elgamal, E. A. Itawi, and A. J. Rao, "Single incision laparoscopic sleeve gastrectomy: a novel technique," Obesity Surgery, vol. 18, no. 10, pp. 1338-1342, 2008.

[31] Y. Otani, M. Ohgami, N. Igarashi et al., "Laparoscopic wedge resection of gastric submucosal tumors," Surgical Laparoscopy and Endoscopy, vol. 10, no. 1, pp. 19-23, 2000.

[32] J. H. Lee, M. S. Lee, H. H. Kim et al., "Comparison of singleincision laparoscopic distal gastrectomy and laparoscopic distal gastrectomy for gastric cancer in a porcine model," Journal of Laparoendoscopic and Advanced Surgical Techniques, vol. 21, no. 10, pp. 935-940, 2011.

[33] S. R. Markar, A. Karthikesalingam, S. Thrumurthy, L. Muirhead, J. Kinross, and P. Paraskeva, "Single-incision laparoscopic surgery (SILS) vs. conventional multiport cholecystectomy: systematic review and meta-analysis," Surgical Endoscopy and Other Interventional Techniques, vol. 26, no. 5, pp. 1205-1213, 2012.
[34] S. Chakravartty, B. Murgatroyd, D. Ashton, and A. Patel, "Single and multiple incision laparoscopic adjustable gastric banding: a matched comparison," Obesity Surgery, vol. 22, no. 11, pp. 16951700,2012 


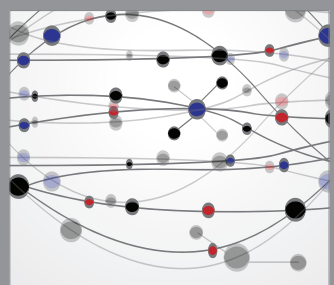

The Scientific World Journal
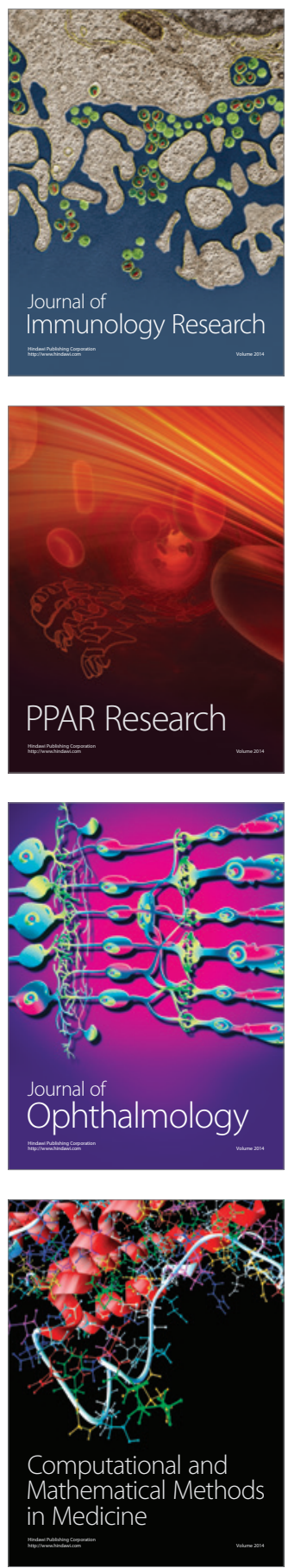

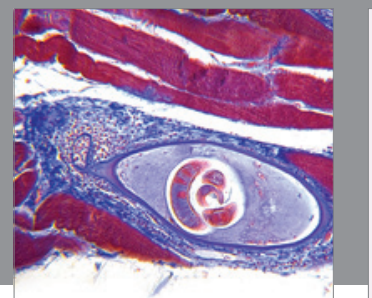

Gastroenterology

Research and Practice
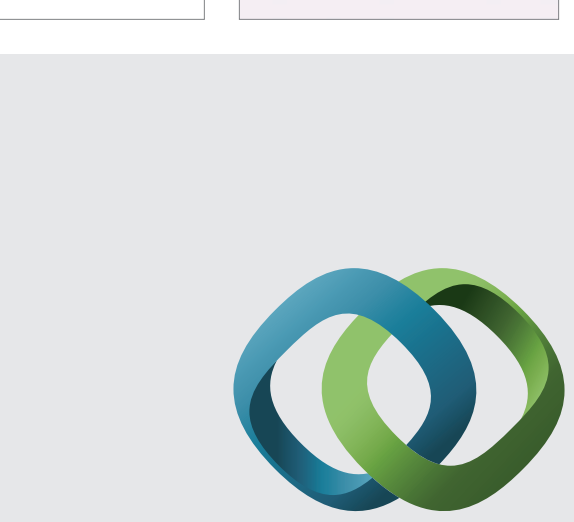

\section{Hindawi}

Submit your manuscripts at

http://www.hindawi.com
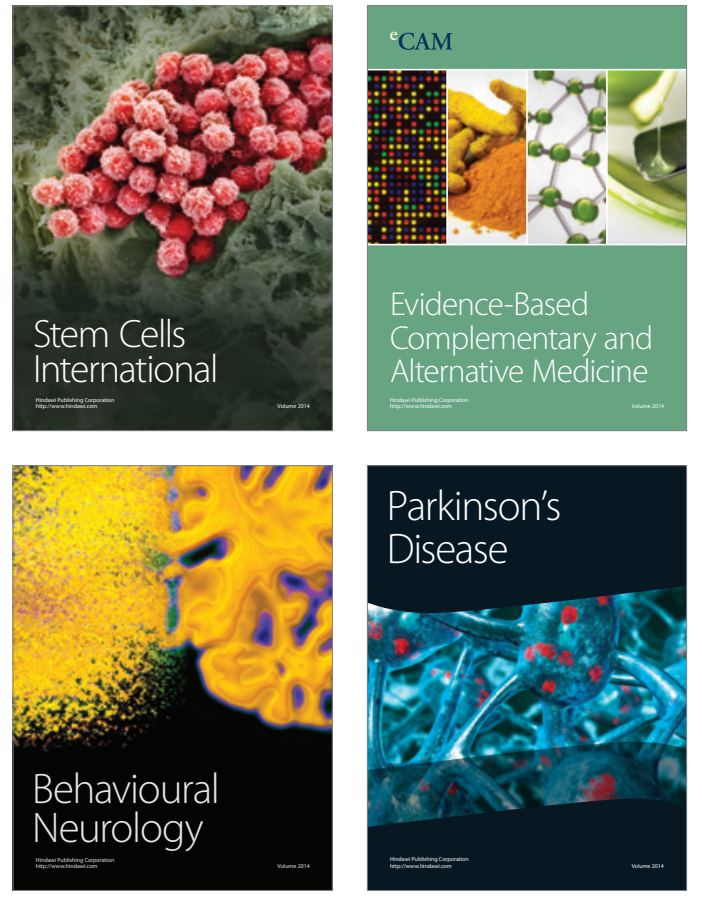
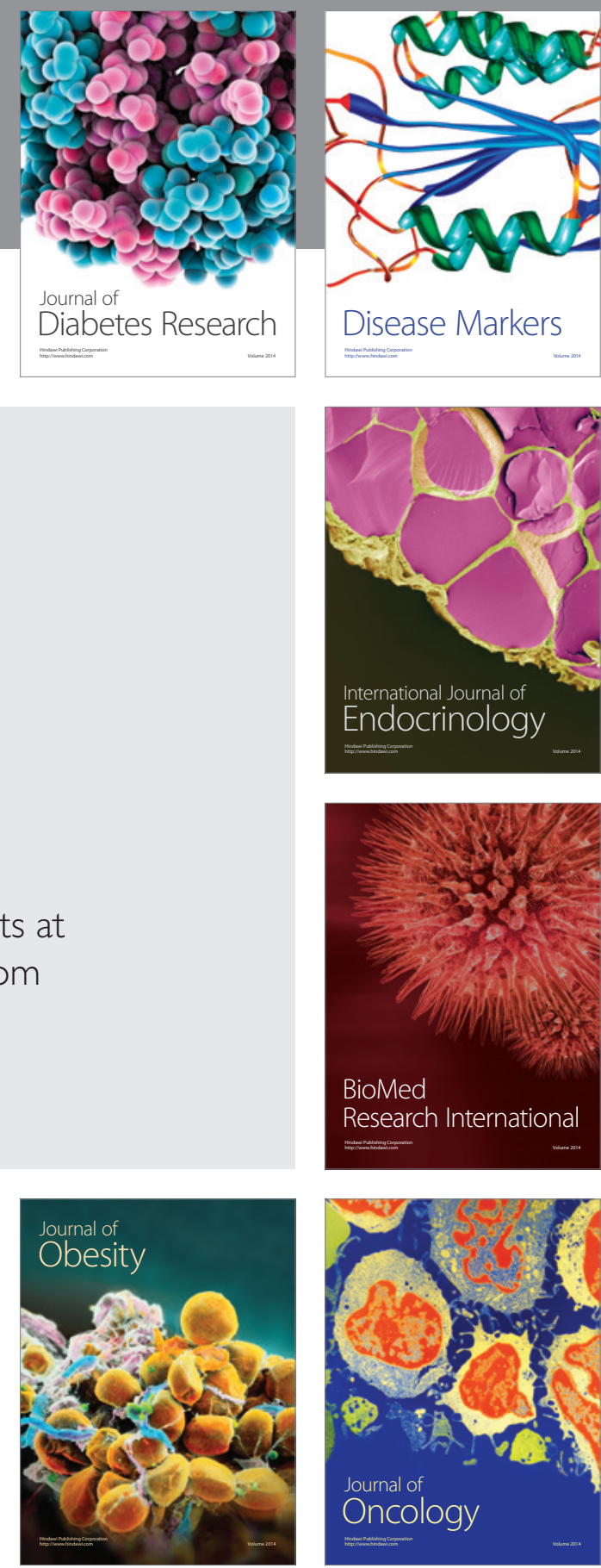

Disease Markers
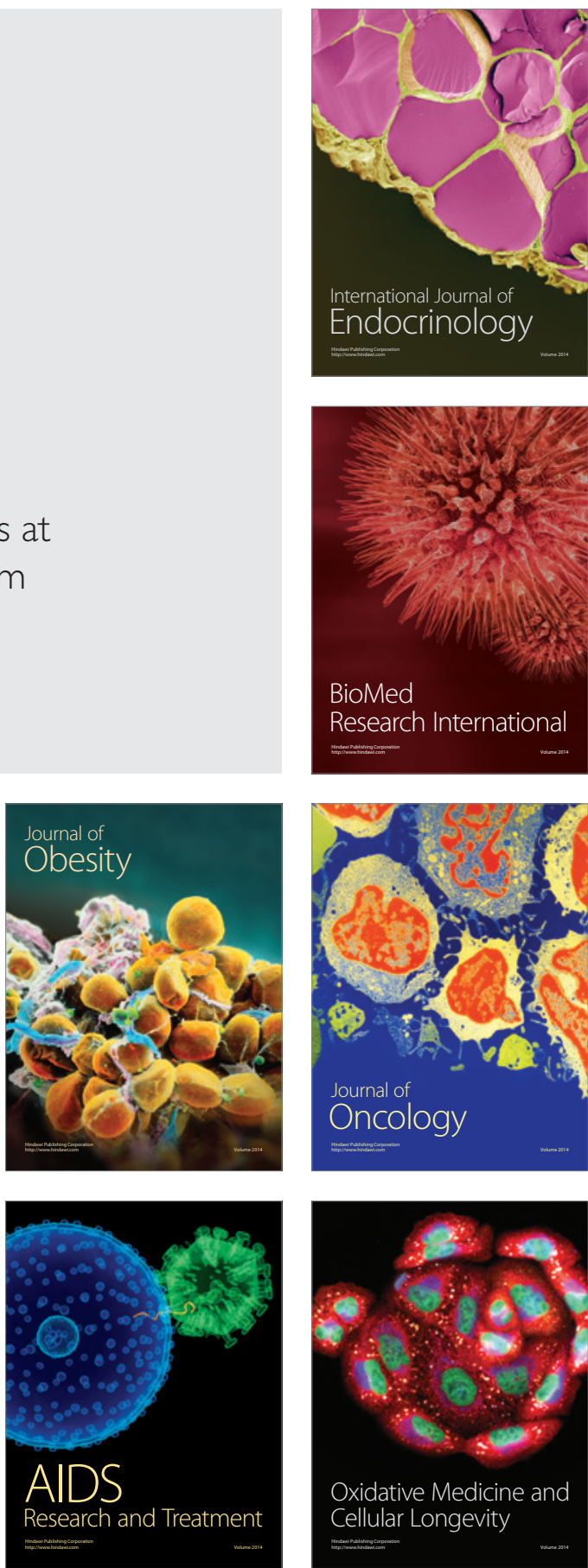